\title{
ENSEÑAR LA CIUDAD A TRAVÉS DE LA HISTORIA, UN ANÁLISIS DE LOS MANUALES ESCOLARES DE CIENCIAS SOCIALES ${ }^{1}$
}

\author{
Teach the City through History, a Social Sciences Textbooks Analysis
}

\author{
Ramón Martínez Medina \\ rmartinez@uco.es \\ Universidad de Córdoba. España
}

Fecha de recepción: 01/03/2019

Fecha de aceptación: 22/06/2019

Resumen: La enseñanza del medio urbano en los últimos años ha cobrado relativa importancia debido a la gran expansión de las ciudades y de la población que habita en ellas a nivel mundial. Sin embargo, como contenido a enseñar no aparece en los Reales Decretos de enseñanzas mínimas de Educación Primaria. Todo ello no desmerece su importancia, pues la ciudad se enseña desde otras perspectivas, fundamentalmente vinculada a la enseñanza de la historia en sus distintos periodos. Este trabajo pretende conocer cómo se enseña la ciudad en los manuales escolares de Ciencias Sociales de Educación Primaria a través del tiempo. La metodología empleada es mixta, cualitativa y cuantitativa. Para el análisis de los manuales se ha diseñado una parrilla con cuatro categorías. En la primera se presentan los datos generales e identificación del manual escolar. En la segunda se analiza la calidad técnica del mismo y la organización interna. La tercera categoría, denominada «La ciudad en el manual escolar», pretende realizar un análisis interpretativo de dichos contenidos a través de las ideas que son vehiculadas y su grado de profundización en el proceso de enseñanza-aprendizaje. También se analiza el tipo de recursos didácticos seleccionados, utilizando la Taxonomía revisada de Bloom. La última categoría tiene un carácter reflexivo, intentando destacar los criterios y principios aplicados para el diseño y la forma en que el manual trata los contenidos sobre la ciudad.

Palabras clave: ciudad; Ciencias Sociales; Historia; manuales escolares; Educación Primaria.

${ }^{1}$ Este trabajo se inscribe en el marzo del Proyecto de investigación PATTERN. Patrimonio Arqueológico, Nuevas Tecnologías, Turismo, Educación y Rentabilización Social: un Nexo Necesario para la Ciudad Histórica, concedido por la Secretaría de Estado de Investigación, Desarrollo e Innovación orientada a los Retos de la Sociedad, convocatoria 2015, Modalidad 1 (HAR2015-68059-C2-1R). 


\begin{abstract}
Teaching the urban environment in recent years has become relatively important due to the great expansion of cities and the population that inhabits them worldwide. However, as content to teach does not appear in the Spanish Primary Education Decree.All thisdoes not detract from its importance, the city is taught from other perspectives,fundamentally linked to the teaching of history in its different periods.This work aims to know how the city is taught in the Social Sciencestextbooks of Spanish Primary Education through history. The methodology used is mixed, qualitative and quantitative.For the textbook'sanalysis, a grid with four categories has been designed.In the first, the general data and identification of the school manual are presented.In the second, technical quality and internal organization are analyzed.The third category, called "The city in the school manual», aims to perform an interpretative analysis of the contents through the ideas that are transmitted and the degree of deepening in the teaching-learning process.The type of didactic resources selected is also analyzed, using Bloom's revised Taxonomy.The last category has a reflexive character, trying to highlight the criteria and principles applied to the design and the way in which the manual deals with the contents about the city.
\end{abstract}

Keywords: city; social science; history; textbooks; primary education.

SUMARIO: 1. Introdución. 2. Objetivo y metodología. 2.1. Objetivo. 2.2. Metodología de análisis. 2.3. Descripción de la muestra. 3. Resultados. 3.1. Resultados del análisis de conceptos y fisionomía de la ciudad. 3.2. Resultados del análisis de los recursos empleados. 4. Conclusiones. 5. Referencias bibliográficas.

\title{
1. INTRODUCIÓN
}

En la actualidad, gran parte de la población mundial vive en ciudades. Según algunas proyecciones de la ONU en los próximos años la población urbana de los países en desarrollo va a duplicarse. Por el contrario, se prevé que la población de los países desarrollados apenas va a aumentar, o incluso disminuir. Según datos del Banco Mundial, el 54 \% de la población reside en núcleos urbanos, pero esta cifra aumenta a más del 75 \% si nos circunscribimos a los países desarrollados del mundo. Así mismo, a medida que la población mundial en desarrollo aumente, el número de ciudades grandes crecerá de manera considerable (Herrero, 2004).

Desde el ámbito de las investigaciones en geografía urbana son muchos los enfoques que las diferentes escuelas de pensamiento han abordado. Estos van desde investigaciones sobre redes y sistemas urbanos, centrando la atención sobre la estructura interna de la ciudad; la adaptación de las ciudades a las condiciones del medio natural y, específicamente, sobre la situación y el emplazamiento; la evolución histórica del espacio urbano; la morfología y los paisajes urbano generados; las investigaciones sobre la trama urbana y las tipologías de vivienda; los usos del suelo en las áreas urbanas, o la validez de los diferentes modelos de organización (modelo concéntrico, sectorial y polinuclear). A estas líneas generales de actuación se añadieron otras sobre la imagen de la ciudad o los mapas mentales, y la cuestión de cómo esto afectan al comportamiento espacial de los ciudadanos (Urteaga y Capel, 1982). 
Teniendo en cuenta la gran cantidad de líneas de investigación existentes sobre la ciudad y su creciente importancia para la población, el estudio de la ciudad ha estado presente desde hace tiempo en el sistema escolar, tanto en los planes de estudio como en las prácticas e innovaciones docentes (González y García, 2014). Su relevancia educativa se justifica porque el alumnado aprende en la ciudad (como entorno o contenedor), de la ciudad (como agente educativo) y aprende la ciudad (como contenido escolar) (Trilla, 1997). Además, las investigaciones desde el campo educativo han puesto en relieve este campo como un potente ámbito de conocimiento escolar bastante flexible que puede permitir un tratamiento interdisciplinar, así como ayudar a diseñar currículos mas coherentes. Todo ello permitiría al alumnado mejorar la comprensión de los fenómenos urbanos, analizar los problemas sociales, ambientales y patrimoniales mas importantes para conocer el funcionamiento de las sociedades y ser capaces de afrontar y resolver como futuros ciudadanos los problemas del mundo actual (García, 2011). Por todo ello, es necesario conocer la evolución histórica de los fenómenos urbanos, pensar históricamente, vinculando el pasado con el presente para ser capaces de afrontar el futuro (García, 2003).

Aunque la legislación vigente de Educación Primaria, Ley para la Mejora de la Calidad educativa (LOMCE) para Ciencias Sociales, no hace referencia explícita sobre la enseñanza de la ciudad y del medio urbano, en las legislaciones autonómicas sí que aparecen referencias a este hecho. Tal es el caso de la comunidad de Andalucía, en la que se enseñan conceptos como medio urbano, o entornos urbanos cercanos para ejemplificar aspectos como la demografía, actividades económicas o modos de vida desde el punto de vista geográfico, o los monumentos, costumbres y tradiciones, desde la perspectiva histórica.

A esto se une una larga tradición de investigaciones que han abordado la enseñanza de la historia a través de los manuales escolares, siendo las más recientes las que analizan las competencias básicas, las actividades o el tipo de imágenes empleadas para construir el conocimiento histórico (Sáiz, 2011; 2013; Bel, 2017 y Bel et al. 2018).

\section{OBJETIVO Y METODOLOGÍA}

\subsection{Objetivo}

El objetivo principal de este trabajo es conocer cómo los manuales escolares, el recurso educativo más frecuentemente utilizado por el profesorado de Educación Primaria, trabajan la ciudad a través de la historia en el sistema educativo español. En concreto, se pretende conocer cómo los manuales de segundo y tercer ciclo de Educación Primaria, adaptados a la normativa de la Ley para la 
Mejora de la Calidad Educativa, trabajan la ciudad en un determinado periodo histórico, a través del análisis de los contenidos, imágenes, texto de autor, así como, y principalmente, del tipo de actividades que se emplean en el proceso de aprendizaje.

\subsection{Metodología de análisis}

Para proceder al análisis de los manuales escolares se ha elaborado un instrumento organizado a partir de la definición de categorías. La parrilla de análisis se ha construido a partir de la utilizada por Cristina Maia (2017), estableciendo cuatro categorías diferentes.

La primera, «identificación del manual escolar», pretende presentar los datos generales de identificación del mismo, especialmente los referidos a la editorial, año de publicación y grado y nivel de educación al que pertenecen. En la segunda categoría de análisis, "caracterización del manual escolar», se pretende analizar la calidad técnica del mismo y la organización interna. El primer parámetro se centra en aspectos relativos al diseño, y el segundo en la estructura del manual, lo que nos permite conocer la coherencia interna del mismo, verificando los siguientes elementos: número de páginas, unidades didácticas, presentación del manual, índice, evaluación inicial, bibliografía, etc.

La tercera categoría, denominada "La ciudad en el manual escolar», pretende realizar un análisis interpretativo del contenido del medio urbano a través de las ideas que son vehiculadas y su grado de profundización en el proceso de enseñanza-aprendizaje de este tema. Para ellos se han considerado los siguientes elementos:

- número de páginas dedicadas al estudio de la ciudad y proporción respecto al manual;

- $\quad$ principales ideas vinculadas al texto del autor;

- los conceptos que se trabajan;

- la presencia de imágenes, diferenciando entre ilustraciones, fotografías, gráficas y tablas;

- el tipo de recursos didácticos seleccionados para el aprendizaje de la ciudad a partir de la taxonomía de Bloom modificada por Costa.

Finalmente, la última categoría, titulada «aspectos que más llaman la atención del análisis del manual», tiene un carácter reflexivo, donde se desarrolla una interpretación personal del manual, intentando destacar los criterios y principios aplicados para el diseño y la forma en que el manual trata los contenidos sobre el medio urbano. 
En cuanto al tipo de recursos didácticos seleccionados, hay que poner de manifiesto que son numerosas las clasificaciones y categorías de actividades existentes (Bloom, 1956; Gallagher y Aschner, 1963; Marzanoy Kendall, 2007; Duarte et al., 2009; Martinha, 2010), que permiten estructurar las actividades de enseñanza y aprendizaje en distintos tipos de demanda cognitiva, que conduzcan, a la vez, a la creación de un pensamiento de distinto orden, del más simple al más superior (Granados, 2017).

La taxonomía de Bloom en el dominio cognitivo (1956), pese a tener más de sesenta años, sigue siendo el marco más utilizado por el profesorado. La taxonomía está relacionada con el aprendizaje, con el dominio del conocimiento. Los objetivos que pretende son diversos: implica la adquisición de nuevo conocimiento, de desarrollo intelectual, de la habilidad y de las actitudes; reconocer hechos específicos, procedimientos, estándares y conceptos que estimulan el desarrollo intelectual constantemente. Estos objetivos se agrupan en seis categorías y se presentan en una jerarquía de complejidad y dependencia, de lo más simple a lo más complejo. Para ascender a una nueva categoría, hay que haber alcanzado un rendimiento adecuado en la anterior, pues cada una utiliza capacidades adquiridas en los niveles anteriores. Las categorías de este dominio son: conocimiento; comprensión; aplicación; análisis; síntesis; y evaluación (Ferraz y Belhot, 2010) (Tabla 1).

Teniendo como base la taxonomía de Bloom, Costa (2005) agrupó las seis categorías en tres niveles de preguntas según la complejidad intelectual. Esta simplificación hace el análisis más sencillo y se centra en un nuevo aspecto: la importancia de las fuentes de comunicación y cómo estas se cruzan para generar el conocimiento propio (Granados, 2017).

Los tres grados descritos por Costa son: recolección de la información, que coincide con los niveles 1 y 2 de Bloom (conocimiento y comprensión), se trata de actividades en las que se desarrolla la habilidad de recordar informaciones y contenidos. El segundo grado es el de procesamiento de la información (nivel 3 aplicación y nivel 4 análisis) mediante actividades de tipo procedimental en las que deben aplicar los conocimientos previamente aprendidos en la categoría anterior. Y finalmente aplicación de la información (niveles 5 y 6 , síntesis y evaluación); este tipo de actividades serían las de mayor complejidad y las que sirven para desarrollar el pensamiento crítico y resolutivo en el alumnado. 
Tabla 1. Estructuración de la Taxonomía de Bloom en el dominio cognitivo.

\begin{tabular}{|c|c|}
\hline Categoría & Descripción \\
\hline \multirow{3}{*}{ 1. Conocimiento } & $\begin{array}{l}\text { Definición: Habilidad de recordar informaciones y contenidos } \\
\text { previamente abordados como hechos, fechas, palabras, teorías, } \\
\text { métodos, clasificaciones, lugares, reglas, criterios, procedimientos, } \\
\text { etc. La habilidad puede implicar recordar una significativa cantidad de } \\
\text { información o hechos específicos. El objetivo principal de esta categoría } \\
\text { nivel es traer a la conciencia esos conocimientos. }\end{array}$ \\
\hline & $\begin{array}{l}\text { Subcategorías: } 1.1 \text { Conocimiento específico: Conocimiento de } \\
\text { terminología; Conocimiento de tendencias y secuencias; } 1.2 \\
\text { Conocimiento de formas y significados relacionados con las } \\
\text { especificidades del contenido: conocimiento de la convención; } \\
\text { Conocimiento de tendencia y secuencia; conocimiento de clasificación } \\
\text { y categoría; Conocimiento de criterio; Conocimiento de metodología; } \\
\text { y } 1.3 \text { Conocimiento universal y abstracción relacionado con un } \\
\text { determinado campo de conocimiento: Conocimiento de principios y } \\
\text { generalizaciones; Conocimiento de teorías y estructuras. }\end{array}$ \\
\hline & $\begin{array}{l}\text { Verbos: enumerar, definir, describir, identificar, denominar, listar, } \\
\text { nombrar, combinar, resaltar, apuntar, recordar, relacionar, reproducir, } \\
\text { solucionar, declarar, distinguir, etiquetar, memorizar, ordenar y } \\
\text { reconocer. }\end{array}$ \\
\hline \multirow{3}{*}{ 2. Comprensión } & $\begin{array}{l}\text { Definición: Habilidad de comprender y dar significado al contenido. } \\
\text { Esta habilidad puede ser demostrada por medio de la traducción del } \\
\text { contenido comprendido para una nueva forma (oral, escrita, diagramas, } \\
\text { etc.) o contexto. En esta categoría, se encuentra la capacidad de } \\
\text { entender la información o el hecho, de captar su significado y de } \\
\text { utilizarla en contextos diferentes. }\end{array}$ \\
\hline & Subcategorías: 2.1 Traslación; 2.2 Interpretación y 2.3 Extrapolación. \\
\hline & $\begin{array}{l}\text { Verbos: cambiar, construir, convertir, decodificar, defender, definir, } \\
\text { describir, distinguir, discriminar, estimar, explicar, generalizar, dar } \\
\text { ejemplos, ilustrar, inferir, reformular, prever, reescribir, resolver, } \\
\text { resumir, clasificar, discutir, identificar, interpretar, reconocer, redefinir, } \\
\text { seleccionar, situar y traducir. }\end{array}$ \\
\hline \multirow{2}{*}{ 3. Aplicación } & $\begin{array}{l}\text { Definición: Habilidad de usar informaciones, métodos y contenidos } \\
\text { aprendidos en nuevas situaciones concretas. Esto puede incluir } \\
\text { aplicaciones de reglas, métodos, modelos, conceptos, principios, leyes } \\
\text { y teorías. }\end{array}$ \\
\hline & $\begin{array}{l}\text { Verbos: aplicar, alterar, programar, demostrar, desarrollar, descubrir, } \\
\text { dramatizar, emplear, ilustrar, interpretar, manipular, modificar, } \\
\text { operacionalizar, organizar, prever, preparar, producir, relatar, resolver, } \\
\text { transferir, usar, construir, esbozar, escribir, operar y practicar. }\end{array}$ \\
\hline
\end{tabular}




\begin{tabular}{|c|c|}
\hline Categoría & Descripción \\
\hline \multirow{3}{*}{ 4. Análisis } & $\begin{array}{l}\text { Definición: Habilidad de subdividir el contenido en partes menores } \\
\text { con la finalidad de entender la estructura final. Esta habilidad puede } \\
\text { incluir la identificación de las partes, el análisis de la relación entre las } \\
\text { partes y el reconocimiento de los principios organizativos involucrados. } \\
\text { Identificar partes y sus interrelaciones. En este punto es necesario no } \\
\text { solo haber comprendido el contenido, sino también la estructura del } \\
\text { objeto de estudio. }\end{array}$ \\
\hline & $\begin{array}{l}\text { Subcategorías: Análisis de elementos; Análisis de relaciones; y Análisis } \\
\text { de principios organizacionales. }\end{array}$ \\
\hline & $\begin{array}{l}\text { Verbos: analizar, reducir, clasificar, comparar, contrastar, determinar, } \\
\text { deducir, diagramar, distinguir, diferenciar, identificar, ilustrar, apuntar, } \\
\text { inferir, relacionar, seleccionar, separar, subdividir, calcular, discriminar, } \\
\text { examinar, probar, esquematizar y cuestionar. }\end{array}$ \\
\hline \multirow{3}{*}{ 5. Síntesis } & $\begin{array}{l}\text { Definición: Habilidad de agregar y juntar partes con el fin de crear un } \\
\text { nuevo todo. Esta habilidad implica la producción de una comunicación } \\
\text { única (tema o discurso), un plan de operaciones (propuestas de } \\
\text { investigación) o un conjunto de relaciones abstractas (esquema para } \\
\text { clasificar informaciones). Combinar partes no organizadas para formar } \\
\text { un «todo». }\end{array}$ \\
\hline & $\begin{array}{l}\text { Subcategorías: } 5.1 \text { Producción de la comunicación original; } 5.2 \\
\text { Producción de un plan o propuestas de un conjunto de operaciones; y } \\
5.3 \text { Derivación de un conjunto de relaciones abstractas. }\end{array}$ \\
\hline & $\begin{array}{l}\text { Verbos: clasificar, combinar, compilar, componer, diseñar, construir, } \\
\text { crear, diseñar, desarrollar, establecer, explicar, formular, generalizar, } \\
\text { inventar, modificar, organizar, originar, planificar, proponer, reorganizar, } \\
\text { relacionar, revisar, reescribir, resumir, sistematizar, escribir, desarrollar, } \\
\text { estructurar, montar y proyectar. }\end{array}$ \\
\hline \multirow{3}{*}{ 6. Evaluación } & $\begin{array}{l}\text { Definición: Habilidad de juzgar el valor del material (propuesta, } \\
\text { investigación, proyecto) para un propósito específico. El juicio se basa } \\
\text { en criterios bien definidos que pueden ser externos (relevancia) o } \\
\text { internos (organización) y pueden ser suministrados o conjuntamente } \\
\text { identificados. Juzgar el valor del conocimiento. }\end{array}$ \\
\hline & $\begin{array}{l}\text { Subcategorías: } 6.1 \text { Evaluación en términos de evidencia interna; y } 6.2 \\
\text { Juicio en términos de criterios externos. }\end{array}$ \\
\hline & $\begin{array}{l}\text { Verbos: Evaluar, averiguar, elegir, comparar, concluir, contrastar, } \\
\text { criticar, decidir, defender, discriminar, explicar, interpretar, justificar, } \\
\text { relatar, resolver, resumir, apoyar, validar, escribir un comentario sobre, } \\
\text { detectar, estimar, juzgar y seleccionar. }\end{array}$ \\
\hline
\end{tabular}

Fuente: Ferraz y Belhot, 2010 a partir de Bloom (1956), Bloom (1986), Driscoll (2000) y Krathwohl (2002). 


\subsection{Descripción de la muestra}

Una vez definido el instrumento se procedió al análisis de las variables establecidas en manuales escolares pertenecientes a las mayores editoriales españolas: Anaya, Edebé, Santillana y SM, integradas en ANELE (Asociación Nacional de Editores de Libros y material de Enseñanza), así como los de Edelvives y Vicens Vives. El conjunto de estas editoriales supone el $90 \%$ del mercado español de libros de texto en Educación Primaria. Todos los libros seleccionados han sido editados con posterioridad a la entrada en vigor de la Ley para la Mejora de la Calidad Educativa (LOMCE). Se han seleccionado libros de Ciencias Sociales de segundo y tercer ciclo, concretamente de los cursos 4. y 5. de Primaria, en los que se enseñan los distintos periodos históricos y además tienen referencias al hecho urbano. En total se han analizado 13 libros de texto. Asimismo, 5 de estos traen de manera complementaria un cuaderno de actividades, que también ha sido analizado. En la tabla 2 se recogen los principales datos identificativos de cada manual.

Tabla2. Datos principales de los manuales analizados

\begin{tabular}{|c|c|c|c|c|c|c|c|c|c|}
\hline Editorial & Título & Año & Curso & ISBN & Código & $\begin{array}{l}\text { N.o } \\
\text { UD }\end{array}$ & $\begin{array}{c}\text { N.o } \\
\text { págs. }\end{array}$ & $\begin{array}{c}\text { C. } \\
\text { Activ. }\end{array}$ & $\begin{array}{c}\text { ISBN } \\
\text { Cuaderno }\end{array}$ \\
\hline \multirow{2}{*}{ Anaya } & $\begin{array}{c}\text { Aprender es } \\
\text { crecer }\end{array}$ & 2015 & 4.. & $\begin{array}{c}978-84-678- \\
7919-3 \\
\end{array}$ & An4 & 6 & 102 & Sí & $\begin{array}{c}978-84-678- \\
7920-9\end{array}$ \\
\hline & $\begin{array}{c}\text { Aprender es } \\
\text { crecer }\end{array}$ & 2015 & 5.ㅇ & $\begin{array}{c}978-84-678- \\
3398-0 \\
\end{array}$ & An5 & 6 & 106 & & \\
\hline \multirow{3}{*}{ Edebé } & $\begin{array}{l}\text { Proyecto Global } \\
\text { Interactivo }\end{array}$ & 2015 & 4.. & \begin{tabular}{|c|}
$978-84-683-$ \\
$2413-5$ \\
\end{tabular} & $\mathrm{Ed} 4$ & 7 & 119 & & \\
\hline & $\begin{array}{c}\text { Global interactive } \\
\text { project }\end{array}$ & 2015 & 4.9 & $\begin{array}{c}978-84-683- \\
2050-2\end{array}$ & Edb4 & 8 & 119 & & \\
\hline & $\begin{array}{c}\text { Global interactive } \\
\text { project }\end{array}$ & 2015 & 5.ㅇ & $\begin{array}{c}\text { 978-84-683- } \\
2294-0\end{array}$ & Edb5 & 7 & 139 & & \\
\hline \multirow{2}{*}{ Edelvives } & $\begin{array}{c}\text { Superpixépolis } \\
\text { SPX }\end{array}$ & 2015 & 4. 9 & $\begin{array}{c}978-84-263- \\
9629-7 \\
\end{array}$ & Ev4 & 6 & 131 & Sí & $\begin{array}{c}978-84-263- \\
9630-3 \\
\end{array}$ \\
\hline & $\begin{array}{c}\text { Superpixépolis } \\
\text { SPX }\end{array}$ & 2015 & 5.ㅇ & $\begin{array}{c}978-84-263- \\
9379-1 \\
\end{array}$ & Ev5 & 6 & 127 & Sí & $\begin{array}{c}978-84-263- \\
9393-7 \\
\end{array}$ \\
\hline \multirow{2}{*}{ Santillana } & Saber Hacer & 2015 & 4.ㅇ & $\begin{array}{c}978-84- \\
8305-571-7\end{array}$ & St4 & 9 & 136 & & \\
\hline & Saber Hacer & 2015 & 5.9 & $\begin{array}{c}978-84- \\
8305-422-2\end{array}$ & St5 & 9 & 148 & & \\
\hline \multirow{2}{*}{ SM } & Savia & 2015 & 4.음 & $\begin{array}{c}978-84-675- \\
7544-6\end{array}$ & Sm4 & 6 & 143 & & \\
\hline & Savia & 2015 & 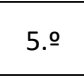 & $\begin{array}{c}978-84-675- \\
7683-2\end{array}$ & Sm5 & 6 & 142 & & \\
\hline \multirow{2}{*}{$\begin{array}{l}\text { Vicens } \\
\text { Vives }\end{array}$} & Aula Activa & 2015 & 4.9 & $\begin{array}{c}978-84-682- \\
3079-5\end{array}$ & Vv4 & 9 & 152 & Sí & $\begin{array}{c}978-84-682- \\
2968-3\end{array}$ \\
\hline & Aula Activa & 2015 & 5.ㅇ & $\begin{array}{c}978-84-682- \\
3139-6\end{array}$ & Vv5 & 9 & 168 & Sí & $\begin{array}{c}978-84-682- \\
1519-8\end{array}$ \\
\hline
\end{tabular}




\section{RESULTADOS}

3.1. Resultados del análisis de conceptos y fisionomía de la ciudad

El análisis del instrumento de recogida de información pone de manifiesto como la enseñanza del hecho urbano sigue la cronología de los acontecimientos históricos. De este modo, las ciudades griegas, cuando aparecen, y romanas se enseñan en segundo ciclo de Educación Primaria, en cuarto curso. Y las relativas al periodo de la Reconquista son abordadas en el ciclo siguiente, en quinto curso (Tabla 3). Como se puede observar, todas las editoriales trabajan la ciudad desde el Imperio romano hasta los Reinos cristianos, no siendo frecuente el estudio del periodo clásico relativo a Grecia, momento de esplendor de las polis con la aparición de la democracia como sistema político. Llama la atención también que en las propuestas didácticas de cursos posteriores no se aborden los cambios acaecidos en las ciudades con el devenir de la Revolución Industrial y la etapa postindustrial que supusieron una enorme transformación de la fisonomía de estas hasta llegar al presente. Esta desconexión temporal impide que durante Educación Primaria se aborden cuáles son las grandes problemáticas actuales de las ciudades como son las desigualdades económicas y sociales, la segregación y marginación de determinados grupos, así como las problemáticas ambientales. Todos estos aspectos, socialmente relevantes e importantísimos para comprender el presente, pasan desapercibidos.

Tabla 3. Contenidos relativos al hecho urbano y periodo histórico

\begin{tabular}{|c|c|c|c|c|}
\hline \multirow{2}{*}{ Código } & \multicolumn{2}{|c|}{ Edad Antigua } & \multicolumn{2}{|c|}{ Edad Media } \\
\hline & Grecia & Roma & Al-Ándalus & Reinos cristianos \\
\hline An4 & & $x$ & & \\
\hline An5 & & & $x$ & $x$ \\
\hline $\mathrm{Ed} 4$ & $x$ & $x$ & & \\
\hline Edb4 & $x$ & $X$ & & \\
\hline Edb5 & & & $x$ & $x$ \\
\hline Ev4 & & $x$ & & \\
\hline Ev5 & & & $x$ & $x$ \\
\hline St4 & $x$ & $\mathrm{x}$ & & \\
\hline St5 & & & $x$ & $x$ \\
\hline Sm4 & & $x$ & & \\
\hline Sm5 & & & $x$ & $x$ \\
\hline Vv4 & & $x$ & & \\
\hline Vv5 & & & $x$ & $X$ \\
\hline
\end{tabular}

Fuente: Elaboración propia. 
La ciudad griega solo es trabajada por tres manuales. En ellos se refleja el concepto de ciudad, qué eran las polis, quiénes eran los ciudadanos y cómo se organizaban en clases sociales según los derechos que cada uno de ellos tenía. En cuanto a los elementos, se pone de manifiesto la ciudad como un espacio amurallado para mantener la seguridad frente a las posibles invasiones y la importancia que otorgan al ágora como espacio de participación y debate. También, existe unanimidad en la transmisión del concepto de acrópolis, como lugar donde se concentra el poder, tanto el divino por la existencia de templos, como el civil, por la existencia de edificios administrativos. El resto de elementos apenas son trabajados, como es el caso del teatro, que sí aparece en todas las propuestas sobre el Imperio romano.

Tabla 4. Conceptos y elementos trabajados vinculados a la ciudad en la época de la Grecia Clásica

Grecia

\begin{tabular}{|c|c|c|c|c|c|c|c|c|c|}
\hline \multirow{2}{*}{ Código } & \multicolumn{4}{|c|}{ Conceptos } & \multicolumn{7}{c|}{ Elementos } \\
\cline { 2 - 11 } & Ciudad & Ciudadanía & $\begin{array}{c}\text { Clases } \\
\text { sociales }\end{array}$ & Ágora & Acrópolis & Templo & Teatro & Estadio & Muralla \\
\hline Ed4 & $\mathrm{X}$ & $\mathrm{X}$ & $\mathrm{X}$ & $\mathrm{X}$ & $\mathrm{X}$ & & & & $\mathrm{X}$ \\
\hline Edb4 & $\mathrm{X}$ & $\mathrm{X}$ & $\mathrm{X}$ & $\mathrm{X}$ & $\mathrm{X}$ & $\mathrm{X}$ & & & \\
\hline St4 & $\mathrm{X}$ & $\mathrm{X}$ & \multicolumn{7}{|c|}{ Fuente: Elaboración propia. } \\
\hline
\end{tabular}

Siguiendo el desarrollo de los manuales de cuarto curso, aparece el Imperio romano. En ellos, el concepto de ciudad no aparece, pero sí la importancia que estas tuvieron en la expansión territorial y como centro de intercambio comercial. Un aspecto que sí es tratado son las clases sociales que existían en el imperio, desde los patricios y plebeyos como ciudadanos libres, hasta los esclavos, personas sin derechos y propiedad de otros.

Tabla 5. Conceptos trabajados vinculados a la ciudad en el Imperio romano

\begin{tabular}{|c|c|c|c|}
\multicolumn{5}{|c|}{ Imperio romano } \\
\hline \multirow{2}{*}{ Código } & \multicolumn{3}{|c|}{ Conceptos } \\
\cline { 2 - 5 } & Ciudad & Ciudadanía & Clases sociales \\
\hline An4 & & $X$ & $X$ \\
\hline Ed4 & & $X$ & $X$ \\
\hline Edb4 & & $X$ & $X$ \\
\hline Ev4 & $X$ & $X$ & $X$ \\
\hline St4 & & & $X$ \\
\hline Sm4 & & $X$ & $X$ \\
\hline Vv4 & & \multicolumn{3}{|c|}{ Fuente: Elaboración propia. } \\
\hline
\end{tabular}


La morfología de las ciudades no es mencionada en ningún manual, no se hace referencia a su planta ortogonal y su origen en muchos casos miliar y solo una de las propuestas menciona el cardo y el decumanus como vías principales. Mayor unanimidad hay con los elementos que existían dentro de ella. Se pueden dividir en dos grandes grupos, por un lado, los destinados al culto y a la socialización como son templos, foro, circo, teatro, anfiteatro y termas. Y por otro, elementos básicos de la ciudad como la muralla que garantizaba la defensa, o los acueductos que servían para el abastecimiento de agua potable. En algunos casos, también aparecen referencias al Arco del Triunfo, como elemento de conmemoración de batallas y exaltación de la figura del emperador.

Tabla 6. Elementos mencionados en la ciudad romana

Imperio romano

\begin{tabular}{|c|c|c|c|c|c|c|c|c|c|}
\hline & \multicolumn{7}{|c|}{ Elementos } \\
\cline { 2 - 10 } Código & Templo & Foro & Circo & Teatro & Anfiteatro & Termas & Muralla & $\begin{array}{c}\text { Arco } \\
\text { del } \\
\text { Triunfo }\end{array}$ & Acueducto \\
\hline An4 & $\mathrm{X}$ & & $\mathrm{X}$ & $\mathrm{X}$ & $\mathrm{X}$ & $\mathrm{X}$ & & & $\mathrm{X}$ \\
\hline Ed4 & $\mathrm{X}$ & $\mathrm{X}$ & $\mathrm{X}$ & $\mathrm{X}$ & $\mathrm{X}$ & $\mathrm{X}$ & $\mathrm{X}$ & & $\mathrm{X}$ \\
\hline Edb4 & $\mathrm{X}$ & & & $\mathrm{X}$ & $\mathrm{X}$ & & & & $\mathrm{X}$ \\
\hline Ev4 & $\mathrm{X}$ & $\mathrm{X}$ & $\mathrm{X}$ & $\mathrm{X}$ & $\mathrm{X}$ & $\mathrm{X}$ & $\mathrm{X}$ & & $\mathrm{X}$ \\
\hline St4 & $\mathrm{X}$ & $\mathrm{X}$ & $\mathrm{X}$ & $\mathrm{X}$ & $\mathrm{X}$ & $\mathrm{X}$ & $\mathrm{X}$ & $\mathrm{X}$ & $\mathrm{X}$ \\
\hline Sm4 & $\mathrm{X}$ & $\mathrm{X}$ & $\mathrm{X}$ & $\mathrm{X}$ & $\mathrm{X}$ & $\mathrm{X}$ & $\mathrm{X}$ & $\mathrm{X}$ & $\mathrm{X}$ \\
\hline Vv4 & $\mathrm{X}$ & $\mathrm{X}$ & $\mathrm{X}$ & $\mathrm{X}$ & $\mathrm{X}$ & $\mathrm{X}$ & $\mathrm{X}$ & $\mathrm{X}$ & $\mathrm{X}$ \\
\hline
\end{tabular}

También, vinculado a la vida cotidiana en la época romana se hace referencia al tipo de viviendas que existían en las ciudades en algunas de las propuestas analizadas. En ellas se puede observar cómo trabajan las diferencias sociales en función del lugar de residencia, dejando patente como los patricios vivían en domus, una vivienda unifamiliar organizada entorno a un patio central. Las personas con menos recursos residían en insulae, edificios construidos con materiales de baja calidad y de varias plantas con viviendas pequeñas, organizados en torno a un patio central. 
Tabla 7. Tipos de viviendas del Imperio romano

Roma

\begin{tabular}{|c|c|c|}
\hline \multirow{2}{*}{ Código } & \multicolumn{2}{|c|}{ Vivienda } \\
\cline { 2 - 3 } & Insulae & Domus \\
\hline An4 & & \\
\hline Ed4 & & \\
\hline Edb4 & & \\
\hline Ev4 & $\mathrm{X}$ & $\mathrm{X}$ \\
\hline St4 & $\mathrm{X}$ & $\mathrm{X}$ \\
\hline Sm4 & $\mathrm{X}$ & $\mathrm{X}$ \\
\hline Vv4 & $\mathrm{X}$ & $\mathrm{X}$ \\
\hline \multicolumn{2}{|c|}{ Fuente: Elaboración propia. } \\
\hline
\end{tabular}

En líneas generales, y salvo la expresión de Vv4, no se realiza una explicación de cada una de las partes de la domus, más allá de su organización en torno al patio. Sin embargo, en las mismas propuestas sí aparece la villa como unidad de explotación del medio agrario, en la que se explica su organización y partes de forma más detallada.

En el siguiente periodo histórico, Edad Media, a diferencia los del curso anterior, todos los de quinto trabajan con mayor o menos grado de detalle los modelos urbanos tanto de Al-Ándalus como de los Reinos cristianos. El abordaje en el periodo islámico pone de manifiesto cómo las ciudades cobraron especial relevancia como centros de intercambios comerciales, por la existencia de talleres y artesanos y por la gran cultura que albergaban en sus bibliotecas, frente a las ciudades cristianas que tendrán su esplendor varios siglos después. En las propuestas analizadas se puede observar como no aparece el concepto ciudad ni tampoco ninguna referencia a los derechos de las personas que habitan en ellas. Sí que subyace en la mayor parte de los casos la segregación de la población en función de sus creencias religiosas, apareciendo áreas específicas y separadas dentro de las medinas para los habitantes que profesaban otra religión, tal es el caso de las juderías o los barrios mozárabes donde residían los cristianos.

También es frecuente que aparezca alguna referencia a la morfología de la ciudad, destacando lo intrincado de sus calles, generalmente estrechas y desordenadas. En cuanto los elementos, todos abordan los conceptos de muralla que rodea la ciudad antigua (medina) en la que se encuentra el alcázar o alcazaba, una fortaleza militar donde residían lo gobernantes. Los espacios de socialización están dominados por el lugar de oración, la mezquita, ampliamente explicado y referenciado, ejemplificado generalmente con la de Córdoba. Además, se mencionan los zocos y los baños como espacios de intercambio de bienes y de relación social. Menos frecuente son las menciones a las alhóndigas, almacén de 
mercancías, o los arrabales, que eran muy frecuentes en todas las ciudades de relativa importancia en la época y que algunos casos llegaron a ser mas grandes que la parte amurallada.

Tabla 8: Elementos mencionados en la ciudad islámica

Al-Ándalus

\begin{tabular}{|c|c|c|c|c|c|c|c|c|}
\hline \multirow{2}{*}{ Código } & \multicolumn{7}{|c|}{ Elementos } \\
\cline { 2 - 10 } & Mezquita & Alcazaba & Zoco & Muralla & Baños & Medina & Alhóndiga & Arrabales \\
\hline An5 & $\mathrm{X}$ & $\mathrm{X}$ & $\mathrm{X}$ & $\mathrm{X}$ & & $\mathrm{X}$ & $\mathrm{X}$ & $\mathrm{X}$ \\
\hline Edb5 & $\mathrm{X}$ & $\mathrm{X}$ & $\mathrm{X}$ & $\mathrm{X}$ & & $\mathrm{X}$ & & \\
\hline Ev5 & & $\mathrm{X}$ & $\mathrm{X}$ & & $\mathrm{X}$ & $\mathrm{X}$ & & \\
\hline St5 & $\mathrm{X}$ & $\mathrm{X}$ & $\mathrm{X}$ & $\mathrm{X}$ & & & & \\
\hline Sm5 & $\mathrm{X}$ & $\mathrm{X}$ & $\mathrm{X}$ & $\mathrm{X}$ & $\mathrm{X}$ & & & \\
\hline Vv5 & $\mathrm{X}$ & $\mathrm{X}$ & $\mathrm{X}$ & $\mathrm{X}$ & $\mathrm{X}$ & $\mathrm{X}$ & $\mathrm{X}$ & $\mathrm{X}$ \\
\hline
\end{tabular}

Fuente: Elaboración propia.

La ciudad medieval, correspondiente a los reinos cristianos en la Edad Media, aparece como un elemento que comienza a resurgir a partir de los siglos XI y XII. La causa de este nuevo esplendor se debe a la aparición de un gran número de artesanos y comerciantes que permitieron que los burgos se expandiesen y apareciera una nueva clase social, la burguesía. En todos los manuales, menos en el An5, que no trabaja la ciudad de forma directa, se abordan las clases sociales, poniendo énfasis en los estamentos, en los que existen grupos privilegiados y otros que no lo son. Se refleja, además, la incapacidad de estos para el ascenso social, aunque pertenezcan a un grupo económicamente poderoso como es la burguesía. También, tratan la aparición de los gremios de artesanos y comerciantes y su tendencia a vivir en los mismos barrios, y encima de los talleres. Respecto a la morfología de la ciudad, ningún manual hace referencia a ella, por lo que el alumnado no sabrá cómo son las calles y su organización interna, no se ejemplifica ningún caso español, como si ocurre con la ciudad de Córdoba para la época islámica. Solo se mencionan algunos elementos distintivos como las murallas y las puertas, los castillos a partir de los cuales surgen estos núcleos y las iglesias o catedrales. Sí se pone de manifiesto la importancia del mercado semanal o de las ferias para el intercambio de todo tipo de productos, especificando, en algunos casos, que solo las ciudades importantes tenían ese privilegio. A veces, se asocia ese mercado al lugar donde de celebraba, generalmente en una plaza mayor donde solía estar ubicado el ayuntamiento o concejo municipal. 
Tabla 9. Elementos mencionados en la ciudad medieval

Reinos cristianos

\begin{tabular}{|c|c|c|c|c|c|c|c|c|}
\hline \multirow{2}{*}{ Código } & \multicolumn{7}{|c|}{ Elementos } \\
\cline { 2 - 10 } & Burgo & Muralla & Puerta & Catedral & $\begin{array}{c}\text { Ayuntamiento } \\
\text { o Concejo }\end{array}$ & Mercado & $\begin{array}{c}\text { Plaza } \\
\text { Mayor }\end{array}$ & Castillo \\
\hline An5 & & & & & & & & \\
\hline Edb5 & & $\mathrm{X}$ & $\mathrm{X}$ & $\mathrm{X}$ & & & $\mathrm{X}$ & \\
\hline Ev5 & $\mathrm{X}$ & & & $\mathrm{X}$ & & $\mathrm{X}$ & & $\mathrm{X}$ \\
\hline St5 & $\mathrm{X}$ & $\mathrm{X}$ & $\mathrm{X}$ & $\mathrm{X}$ & $\mathrm{X}$ & $\mathrm{X}$ & & \\
\hline Sm5 & $\mathrm{X}$ & & & $\mathrm{X}$ & & $\mathrm{X}$ & & \\
\hline Vv5 & & $\mathrm{X}$ & $\mathrm{X}$ & $\mathrm{X}$ & $\mathrm{X}$ & $\mathrm{X}$ & $\mathrm{X}$ & $\mathrm{X}$ \\
\hline
\end{tabular}

Por lo expuesto anteriormente, en cuanto a los textos de autor, podemos observar como el concepto de ciudad solo es tratado en algunos manuales de cuarto curso referenciándolo al nacimiento de estas en época griega. Después ese concepto no es trabajado en los demás periodos históricos, aunque sería conveniente recordarlo, sobre todo en el curso siguiente. Tampoco hay referencias a la forma de las ciudades y a su organización interna, salvo en el caso de la ciudad islámica. Se puede afirmar que el estudio de la ciudad se realiza a través del análisis de algunos de los elementos más distintivos, pero no en su conjunto, como un elemento vivo en el que se han ido superponiendo las diferentes civilizaciones hasta llegar a la apariencia actual contemporánea. Sí que queda clara y patente la aparición de otros conceptos relativos a la ciudadanía, a las clases sociales, que son mucho mejor explicadas, así como los derechos o el papel que a cada clase o estamento le correspondían. Por último, hay que destacar la ruptura que supone no continuar la enseñanza del medio urbano hasta la actualidad, siguiendo el devenir histórico que introducen los libros, del resto de unidades de quinto curso y de sexto en los que se abordan la Edad Moderna y la Contemporánea. En todas las propuestas analizadas para quinto no existe ninguna referencia posterior, y en los libros consultados de sexto curso solo se hace referencia a la ciudad industrial en la propuesta de Vicens Vives, una de las editoriales que mejor trabaja esta temática a partir de la sección Vivo en...

También queda patente la desconexión existente con los problemas sociales relevantes de la época. Si bien, es cierto que se incluyen aspectos relativos a las diferencias sociales o a la segregación residencial en función de la clase, estas se abordan de manera somera. Además, existe una total desconexión con las problemáticas sociales actuales, lo que serviría para ejemplificar que muchos de estos problemas del pasado siguen vigentes en el presente. Esto ayudaría mucho a dotar de funcionalidad y hacer atractiva la enseñanza y aprendizaje de las Ciencias Sociales para los discentes en la era de la tecnología y la globalización. 


\subsection{Resultados del análisis de los recursos empleados}

En cuanto al análisis de los recursos o actividades empleados para la enseñanza de la ciudad en los diferentes manuales hay que poner de manifiesto en primer lugar que son escasos, una media de 11 actividades por libro, debido a la reducida presencia que este contenido tiene, que no suele superar las dos páginas para cada periodo histórico. Solo en los casos en que se ha podido analizar también el cuadernillo de actividades el número de estas es más elevado.

Como se ha explicado anteriormente, se analizaron los diferentes manuales siguiendo la taxonomía de Bloom. Las actividades recogidas en los diferentes libros de texto se categorizaron según la complejidad de procesos cognitivos: 1 (conocimiento), 2 (comprensión), 3 (aplicación), 4 (análisis), 5 (síntesis) y 6 (evaluación). A modo de ejemplo se ha elaborado una tabla con actividades que corresponden a cada una de las tipologías (Tabla 10).

Tabla 10. Ejemplo de actividades según la taxonomía de Bloom.

\begin{tabular}{|c|c|l|}
\hline $\begin{array}{c}\text { Taxonomía } \\
\text { de Bloom }\end{array}$ & Manual & \multicolumn{1}{c|}{ Actividad } \\
\hline 1 & Ev4 & $\begin{array}{l}\text { ¿Cómo se llama la construcción que llevaba el agua hasta las } \\
\text { ciudades? }\end{array}$ \\
\hline 2 & St5 & $\begin{array}{l}\text { Describe la ciudad andalusí: ¿Cómo se protege? ¿Cómo es el interior? } \\
\text { ¿Cómo son las calles? }\end{array}$ \\
\hline 3 & Ev5 & $\begin{array}{l}\text { En base a las imágenes dadas, escribe el nombre de estos lugares de } \\
\text { una ciudad medieval. Después, indica su función principal. }\end{array}$ \\
\hline 4 & Vv4 & Explicas las diferencias entre dos tipos de viviendas (domus e insula). \\
\hline 5 & An4 & Observa la imagen de una ciudad romana y redacta lo que te sugiera. \\
\hline 6 & Vv5 & $\begin{array}{l}\text { Averigua qué restos de la antigua Córdoba califal existen todavía en } \\
\text { la Córdoba actual. }\end{array}$ \\
\hline
\end{tabular}

Fuente: Elaboración propia.

De manera simultánea se tuvo en consideración los 3 niveles de cuestionamiento de Arthur Costa, de modo que las actividades 1 y 2 pertenecen a actividades de introducción al conocimiento (conceptuales), las 3 y 4 son de tipo aplicación de conocimiento (procedimentales en muchos casos), y 5 y 6 son de dominio y maestría del conocimiento tratado (adquisición de competencias). La siguiente tabla recoge el número de actividades de cada tipo en cada manual (Tabla 11). Además, se recoge el porcentaje actividades de cada categoría respecto del total. 
Tabla 11. Número y porcentaje de actividades clasificadas en las categorías de la Taxonomía de Bloom

\begin{tabular}{|c|c|c|c|c|c|c|c|c|c|c|c|c|c|}
\hline \multirow{2}{*}{ Código } & \multicolumn{10}{|c|}{ Categoría } \\
\cline { 2 - 15 } & $\mathbf{1}$ & $\mathbf{\%}$ & $\mathbf{2}$ & $\mathbf{\%}$ & $\mathbf{3}$ & $\mathbf{\%}$ & $\mathbf{4}$ & $\mathbf{\%}$ & $\mathbf{5}$ & $\mathbf{\%}$ & $\mathbf{6}$ & $\mathbf{\%}$ & Total \\
\hline An4 & 5 & 62,5 & 1 & 12,5 & 0 & 0,0 & 1 & 12,5 & 1 & 12,5 & 0 & 0,0 & 8 \\
\hline An5 & 1 & 33,3 & 1 & 33,3 & 0 & 0,0 & 0 & 0,0 & 0 & 0,0 & 1 & 33,3 & 3 \\
\hline Ed4 & 1 & 33,3 & 2 & 66,7 & 0 & 0,0 & 0 & 0,0 & 0 & 0,0 & 0 & 0,0 & 3 \\
\hline Edb4 & 0 & 0,0 & 0 & 0,0 & 0 & 0,0 & 0 & 0,0 & 0 & 0,0 & 0 & 0,0 & 0 \\
\hline Edb5 & 2 & 33,3 & 3 & 50,0 & 1 & 16,7 & 0 & 0,0 & 0 & 0,0 & 0 & 0,0 & 6 \\
\hline Ev4 & 13 & 56,5 & 7 & 30,4 & 1 & 4,3 & 1 & 4,3 & 0 & 0,0 & 1 & 4,3 & 23 \\
\hline Ev5 & 1 & 16,7 & 2 & 33,3 & 2 & 33,3 & 0 & 0,0 & 0 & 0,0 & 1 & 16,7 & 6 \\
\hline St4 & 4 & 28,6 & 8 & 57,1 & 0 & 0,0 & 0 & 0,0 & 0 & 0,0 & 2 & 14,3 & 14 \\
\hline St5 & 6 & 33,3 & 10 & 55,6 & 1 & 5,6 & 0 & 0,0 & 0 & 0,0 & 1 & 5,6 & 18 \\
\hline Sm4 & 2 & 22,2 & 3 & 33,3 & 2 & 22,2 & 1 & 11,1 & 0 & 0,0 & 1 & 11,1 & 9 \\
\hline Sm5 & 3 & 42,9 & 1 & 14,3 & 0 & 0,0 & 1 & 14,3 & 1 & 14,3 & 1 & 14,3 & 7 \\
\hline Vv4 & $\mathbf{7}$ & 33,3 & 5 & 23,8 & 2 & 9,5 & 5 & 23,8 & 0 & 0,0 & 2 & 9,5 & 21 \\
\hline Vv5 & 14 & 43,8 & 11 & 34,4 & 2 & 6,3 & 1 & 3,1 & 1 & 3,1 & 3 & 9,4 & 32 \\
\hline Total & 59 & 39,3 & 54 & 36,0 & 11 & 7,3 & 10 & 6,7 & 3 & 2,0 & 13 & 8,7 & 150 \\
\hline
\end{tabular}

Fuente: Elaboración propia.

Los valores arrojados en esta tabla permiten evaluar la cantidad y complejidad cognitiva de las actividades de cada manual. Para el caso de aquellos manuales con cuaderno de actividades, debe mencionarse que la discusión del análisis realizado se lleva a cabo de manera conjunta (teniendo en cuenta tanto las actividades del libro como las del cuaderno). Dicho esto, se puede observar que existen diferencias en cuanto a la cantidad y variedad (en cuanto al nivel cognitivo) en cada manual, por lo que resulta más conveniente relatar los aspectos más significativos de cada libro.

El manual An4 presenta un total de 8 actividades, siendo el aspecto más relevante la falta de variedad de actividades. No recoge ninguna actividad de las categorías 3 y 6 , y más de la mitad son del grupo 1 (conceptuales), el menos complejo cognitivamente. El $75 \%$ de las actividades son de bajo nivel cognitivo (1-2-3), frente al $25 \%$ de las de alto nivel (4-5-6). El manual An5, aunque cuenta con un número mucho más reducido de actividades, tan solo 3 , estas son variadas y de diversa complejidad, aunque continúan teniendo un importante valor las de mayor facilidad (67\%).

El manual Ed4, como el anterior, solo presenta 3 actividades, siendo todas de baja complejidad (categorías 1 y 2). Mientras que el siguiente, el Edb4, de la misma editorial, pero en versión bilingüe, no contiene ninguna actividad para la temática 
estudiada en este trabajo. Hay que destacar que este libro apenas cuenta con actividades, por lo que debe tratarse de un complemento a la versión en castellano. La otra propuesta de la misma editorial para quinto curso, Ed5, presenta un total de 6 actividades, todas ellas de bajo nivel cognitivo, entre las categorías 1 a 3, conceptuales y procedimentales.

El libro Ev4 es el segundo que presenta mayor cantidad de actividades, un total de 23, con una amplia distribución, ya que, como puede observarse, existe al menos una de cada categoría, menos en la 5 . No obstante, la proporción de tipo 1 es muy elevada (56,5 \%). En cuanto al balance de actividades de bajo nivel cognitivo (1-2-3) versus alto nivel cognitivo (4-5-6), está en una relación $90 \%-10 \%$. Similar ocurre con la propuesta Ev5, con tan solo 6 actividades, el recuento en cuanto a niveles cognitivos es similar, aunque algo más favorable que el anterior, 84 \%-16 \%.

Las dos propuestas de Santillana, St4 y St5, presentan en conjunto un número de actividades similar, 14 y 18 respectivamente, con una estructura en cuanto a nivel cognitivos también parecida. No presentan ejercicios de las categorías 4 y 5 , mientras que entre las tres primeras alcanzan el $85 \%$ y $82 \%$ respectivamente.

En las propuestas de la editorial SM, Sm4 y Sm5, destaca la alta distribución por categorías cognitivas, aun siendo manuales que no superan las diez actividades cada uno. La categoría de actividades con más presencia en el Sm5 es la 1, mientras que en el Sm4 es la 2, actividades en las que los procesos cognitivos empiezan a ser más complejos. La proporción de actividades (1-2-3) vs (4-5-6) arroja un valor diferente, si bien en Sm4 destacan las primeras (77 \% a $22 \%$ ), en el Sm5 la proporción es mucho más equilibrada ( $57 \%$ a $43 \%$ ), siendo este manual el que presenta una mejor proporción en cuanto a complejidad de las actividades.

Lo más destacable de las propuestas de Vicens Vives es el elevado número de actividades existentes, contando además con un apartado específico para el estudio de los núcleos de población y ciudades en función de la época histórica de la que se trate. También tienen un cuaderno de actividades bastante amplio que permite reforzar lo aprendido en el manual. En lo que a la distribución de categorías de Bloom se refiere, el manual Vv4 muestra una estructurade actividades más o menos equilibrada en cuanto a la clasificación de mayor o menor complejidad (67\% frente a $33 \%)$, lo que lo sitúa como la segunda propuesta más equilibrada tras el Sm5. Por último, el Vv5 es la propuesta que mejor y más ampliamente trabaja la ciudad, presenta un total de 32 actividades, por encima de todos los demás manuales. Pero, pese a ello, casi todas ellas son de baja complejidad cognitiva, concretamente el $84 \%$ del total.

En cuanto al análisis de los niveles de cuestionamiento de Arthur Costa de las actividades, la siguiente tabla, similar a la anterior, da luz sobre el número de actividades y proporción en cada nivel 1, 2, 3. Es destacable que todos casos analizados presentan una elevada proporción de actividades de introducción al conocimiento, nivel 1 (conceptuales), superior al $50 \%$. Incluso, 6 de los 13 
manuales presentan un valor superior al $70 \%$ de en este nivel. Por otro lado, respecto a la proporción de actividades de nivel 2 (puesta en práctica del conocimiento aprendido, actividades de tipo procedimental) el análisis muestra una proporción máxima del $33 \%$, siendo lo más frecuente proporciones inferiores al $12 \%$ e incluso inexistentes. Respecto al nivel 3, actividades en las que se debe demostrar dominio y maestría de los conocimientos aprendidos, encontramos 2 grupos de manuales. Aquellos que recogen una proporción muy baja (inferior al $16 \%$ ) de estas actividades (la mayoría) y otro cuyos rangos están por encima del $20 \%$ (An5 y Sm5).

Finalmente, y de manera global, si atendemos al total de actividades de todos los manuales, la proporción de actividades del nivel 1 (introducción al conocimiento) está cercana al $78 \%$, frente a un $9 \%$ de las de aplicación del conocimiento y un $12 \%$ de las de dominio y maestría del conocimiento. Ello denota la excesiva presencia, en términos generales, de actividades básicas en las que solo se trabajan los conceptos, y a su vez, la escasez de actividades de demostración de dominio del tema.

Tabla 11. Número y porcentaje de actividades según su nivel decuestionamiento (según Arthur Costa)

\begin{tabular}{|c|c|c|c|c|c|c|}
\hline \multirow{2}{*}{ Código } & \multicolumn{7}{|c|}{ Categoría } \\
\cline { 2 - 7 } & $\mathbf{1}$ & $\mathbf{\%}$ & $\mathbf{2}$ & $\mathbf{\%}$ & $\mathbf{3}$ & $\mathbf{\%}$ \\
\hline An4 & 6 & 75,0 & 1 & 12,5 & 1 & 12,5 \\
\hline An5 & 2 & 66,7 & 0 & 0,0 & 1 & 33,3 \\
\hline Ed4 & 3 & 100,0 & 0 & 0,0 & 0 & 0,0 \\
\hline Edb4 & 0 & 0,0 & 0 & 0,0 & 0 & 0,0 \\
\hline Edb5 & 5 & 83,3 & 1 & 16,7 & 0 & 0,0 \\
\hline Ev4 & 20 & 87,0 & 2 & 8,7 & 1 & 4,3 \\
\hline Ev5 & 3 & 50,0 & 2 & 33,3 & 1 & 16,7 \\
\hline St4 & 12 & 85,7 & 0 & 0,0 & 2 & 14,3 \\
\hline St5 & 16 & 88,9 & 1 & 5,6 & 1 & 5,6 \\
\hline Sm4 & 5 & 55,6 & 3 & 33,3 & 1 & 11,1 \\
\hline Sm5 & 4 & 57,1 & 1 & 14,3 & 2 & 28,6 \\
\hline Vv4 & 12 & 57,1 & 7 & 33,3 & 2 & 9,5 \\
\hline Vv5 & 25 & 78,1 & 3 & 9,4 & 4 & 12,5 \\
\hline Total & 113 & 75,3 & 21 & 14,0 & 16 & 10,7 \\
\hline
\end{tabular}

Fuente: Elaboración propia. 


\section{CONCLUSIONES}

La presente investigación ha puesto de manifiesto cómo se enseña la ciudad a través la historia en los manuales escolares de Educación Primaria. Se ha podido comprobar como esta no es lineal, ni continua en el tiempo. Desde Grecia hasta la ciudad medieval sí existe una sucesión, pero desde ese momento hasta la actualidad no aparece ninguna referencia, lo que impide que el alumnado pueda comprender el presente si desconoce algunos aspectos del pasado relevantes. En líneas generales se ha puesto de manifiesto la ausencia del concepto de ciudad, así como las grandes carencias existentes en cuanto a la localización, morfología y estructura urbana. Además, no abordan los problemas sociales relevantes de las ciudades, ni los del pasado ni los del presente. Se echa en falta trabajar cuestiones relevantes como son la desigualdad social y las diferencias económicas, o la segregación de la población, ya sea por motivos económicos o religiosos. Estas cuestiones, socialmente vivas en la actualidad, vinculadas con los problemas urbanos actuales, permitirían vehicular una enseñanza de las Ciencias Sociales dotada de funcionalidad y significado, para crear ciudadanos comprometidos y activos, que sean capaces de ver las injusticias y los problemas del mundo en el que viven.

En general, el número de actividades propuestas de esta temática son escasas, salvo alguna excepción. Los recursos empleados para las mismas son mayoritariamente fotos o dibujos, quedando de lado cualquier referencia a la cartografía o los planos, a los que tan acostumbrados están desde la generalización de las nuevas tecnologías de la información y la comunicación. Además, en lo que se refiere a la tipología de actividades teniendo en cuenta la taxonomía de Bloom, se observa una presencia excesiva de actividades cognitivamente sencillas y una carencia en actividades complejas. Esta idea queda reforzada si atendemos al análisis realizado teniendo en cuenta los niveles de cuestionamiento de Costa, ya que se observan las proporciones más altas para las actividades de introducción a los conceptos y, por el contrario, la escasa presencia de actividades de maestría y dominio de los contenidos.

Frente a lo afirmado en el trabajo, conviene señalar algunas limitaciones existentes como son el hecho de no conocer cómo estas actividades son abordadas por los docentes en el aula, ni si en otros territorios se cuenta con el mismo tipo de materiales. Por todo ello, conviene recordar que los manuales escolares no dejan de ser un recurso más al alcance del docente y por tanto desde su práctica se deben desarrollar estrategias educativas que permitan ampliar y complejizar la visión que el alumnado tiene del mundo que lo rodea, para que sean capaces de conocer tanto las causas y como las consecuencias de lo que ocurre en nuestro entorno, tanto pasado como presente. 


\section{REFERENCIAS BIBLIOGRÁFICAS}

Bel, J. C. (2017). Imagen y libros de texto de Historia en Educación Primaria: estudio comparativo a partir de un análisis cualitativo. Revista de Educación, 377, pp. 82-112.

Bel, J. C., Colomer, J. C. y Valls, R. (2019). Alfabetización visual y desarrollo del pensamiento histórico: actividades con imágenes en manuales escolares. Educación XX1, 22(1), pp. 353-374

Bloom, B. S. (1956). Taxonomy of Educational Objectives: The classification of educational goals. Handbook I. Cognitive domain. Nueva York: Longman.

Bloom B. S. (1986). What we are learning about teaching and learning: a summary of recent research. Principal, 66(2), pp. 6-10.

Costa, A. (2005). Arthur Costa's levels of questions [enlínea]. Springfield Public Schools. <www.sps186.org/downloads/basic/274780/Costa\%20and\%20Blooms.pdf>.

Ferraz A. P. y Belhot R. (2010). Taxonomia de Bloom: revisão teórica e apresentação das adequações do instrumento para definição de objetivos instrucionais. Gestão \& Produção, 17(2), pp. 421-431.

Driscoll,M. (2000). Psychology of learning for instruction. Needhan Heights: Allyn \& Bacon.

Duarte, J., Claudino, S., Silva, C., Santo, E. y Carvalho, L. (2009). Podem os manuais escolares contribuir para a melhoria da escola? En A. Antonio, E. Estrela, C. Galego y A. Teodoro (Eds.), Educando o Cidadão Global. Globalização, Educação e Novos Modos de Governação (pp. 578-598). Lisboa, Edições Universitárias Lusófonas.

Gallagher, J. y Aschner, M. J. (1963). A preliminary report on analysis of classroom interaction. Merrill-Palmer Quarterly of Behaviour and Development, 9, pp. 183-194.

García, F. F. (2003). Investigando los asentamientos humanos: la problemática del medio urbano. Investigación en la escuela, 51, pp. 55-69. 
García, F. F. (2011). Problemas del mundo y educación escolar: un desafío para la enseñanza de la Geografía y las ciencias sociales. Revista Brasileira de Educação em Geografia, 1(1), pp. 108-122

González J. F. y García-Pérez F. F. (2014). Las concepciones de los alumnos sobre el medio urbano y sus implicaciones didácticas. Un estudio en Bogotá. Didáctica geográfica, 15, pp. 41-60.

Granados, J. (2017). La formulación de buenas preguntas en didáctica de la geografía. Documents d'Anàlisi Geogràfica, 63(3), pp. 545-559.

Maia C. (2017). A investigação em Manuais Escolares e suasdimensões analíticas: exemplo de umestudo comparativo europeu. Indagatio Didáctica, 9(3), pp. 7-24.

Martinha, C. (2010). Inquéritos a Professores (Estagiários) de Geografia: umareflexão metodológica e umaoportunidade de reflexão e de desenvolvimento de práticas de ensino em Didáctica das CiênciasSociais. Cadernos do Doutoramento em Geografia, 2, pp. 87-115.

Marzano, R. y Kendall, J. (2007). The new taxonomy of educational objectives. 2. ed. CA, Thousand Oaks: Corwin.

Krathwohl, D. R. (2002). A Revision of Bloom's Taxonomy: An Overview. Theory into Practice, 41(4), pp. 212-218

Sáiz, J. (2011). Actividades de libros de texto de Historia, competencias básicas y destrezas cognitivas, una difícil relación: análisis de manuales de 1.0 y 2.0 de ESO. Didáctica de las ciencias experimentales y sociales, 25, pp. 37-64.

Sáiz, J. (2013). Alfabetización histórica y competencias básicas en libros de texto de historia y aprendizajes de estudiantes. Didáctica de las ciencias experimentales y sociales, 27, pp. 43-66.

Trilla, J. (1997). La educación y la ciudad. Revista del Instituto para la Investigación y el Desarrollo Pedagógico, 2, pp. 7-19.

Urteaga, L. y Capel, H. (1982). La geografía y la didáctica del medio urbano. Revista de geografía, 16-17(1), pp. 113-125. 